\title{
Data processing of magnetotelluric survey data using maximum entropy method and IIR filter
}

\author{
Hikaru NAGATA $^{1}$, Hitoshi MIKADA ${ }^{1}$, Tada-nori GOTO ${ }^{1}$, \\ Junichi TAKEKAWA ${ }^{1}$ and Takafumi KASAYA ${ }^{2}$ \\ ${ }^{1}$ Dept. of Civil and Earth Res. Eng., Kyoto University \\ ${ }^{2}$ JAMSTEC.
}

\begin{abstract}
Data processing of magnetotelluric (MT) survey has been applied frequency by frequency based on the Fast Fourier Transform (FFT), since the FFT gives us the response functions (RFs) of the earth in the frequency domain directly. However, applying FFT processing to MT data may not be optimum. It is because the MT data is in general non-stationary since the source of MT is the transient fluctuation of electric current in the ionosphere. As well known, the FFT assumes time series to be a stationary so that we develop the data processing without FFT. We focus on an IIR filter called 'pole on pedestal' that extracts the signal at a specific frequency. Combining this IIR filter and the Hilbert transform, the RFs are calculated in time domain. In addition, it is important to remove the time segments contaminated by noise out of the whole recorded time series before calculating the RFs. Several coherences (for example, partial or multiple coherences) have usually been used to remove the segments contaminated by noise. However, it is known that these coherences are insufficient to select the segments. We apply the maximum entropy method (MEM) to the selection of the segments contaminated by large noise. The MEM searches and removes these contaminated segments easily. As a result we developed the time domain processing of MT data using MEM and IIR filter, and applied this processing to the real data acquired at the Nankai trough. Comparing the conventional and novel data processing, our novel data processing gives us more optimum RFs than the conventional processing.
\end{abstract}

\section{Introduction}

Data processing is one of the essential techniques to obtain optimum response functions (RFs) of the earth. The processing of magnetotelluric (MT) survey data is based on the spectrum analysis using the Fast Fourier Transform (FFT). The robust processing based on FFT has been used widely ${ }^{1}$. This processing gives us optimum RFs of the earth when the $\mathrm{S} / \mathrm{N}$ ratio is high and the time window length is long enough. However, the errors of response function become very large when the data quality is low or the length of time-series is short. This reason is that MT data is in general non-stationary since the source of MT is the transient electromagnetic fluctuation in the ionosphere. On the other hand, FFT assumes input time series to be stationary. Therefore, when the duration of input signal is short, we do not get optimum response function since the stationary assumption of FFT does not satisfy. In order to solve this problem, we developed the data processing without FFT by using a IIR filter. In addition, it is very important for the data processing of MT to select the analyzed segments or remove the segments contaminated by noise. Robust and coherence sort algorithm have been developed and applied in order to select the segments contaminated by noise. However, these methods are sometimes insufficient to remove the contaminated segments. We developed the segments selection algorithm by using MEM. We apply our novel data processing to the real data acquired at Nankai trough and compared to the conventional method.

\section{METHOD}

\subsection{POLE ON PEDESTAL}

We focus on an IIR filter to calculate the RFs in time domain without FFT. This IIR filter is called 'pole on pedestal' 2) that extracts and amplifies signals at a specific frequency. Pole on pedestal defines $Z$ transform (Eq.1).

$$
\mathrm{H}(\mathrm{z})=\frac{\mathrm{SZ}_{0}-Z}{p Z_{0}-Z} \cdot \frac{\mathrm{S} \bar{Z}_{0}-\bar{Z}}{p \bar{Z}_{0}-\bar{Z}}
$$

There is the opposite relation between pole on pedestal filter and notch filter. Eq.1 is defined by multiplying two transfer functions whose relation is time reversal, so that we can make zero phase filter. $\mathrm{S}$ and $\mathrm{q}$ are the locations of pole and zero point on 
the $\mathrm{Z}$ plane. Fig.1 shows an example of applying the pole on pedestal to the real electric field data. Upper figure shows the raw data. We applied the pole on pedestal to this data and extracted the signal

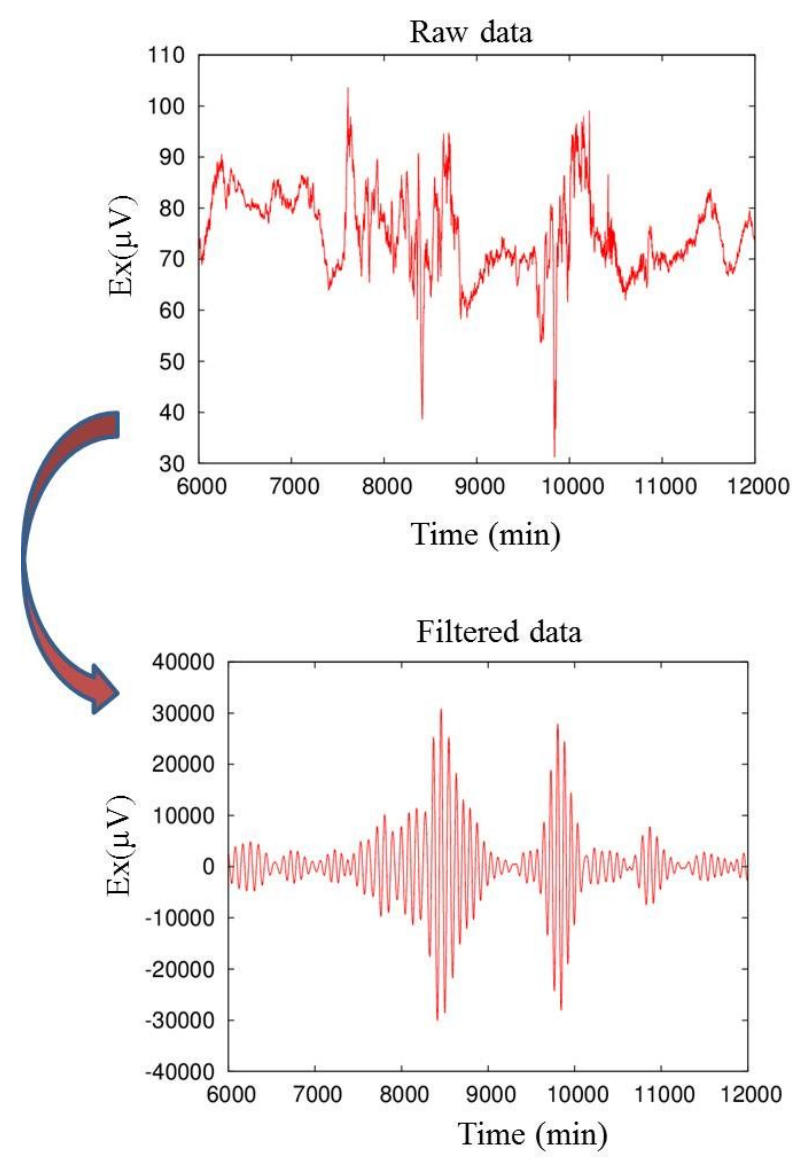

Figure 1 An example of applying the pole on pedestal to the real electric field data. Left figure shows raw data and right figure shows filtered data.

at 5000 sec. After filtering, the wave length is constant and the attenuation of the signal is shown.

\subsection{CALCULATE RFs IN TIME DOMAIN}

Applying pole on pedestal filter, we can get the signal at a specific frequency. The extracted signal has the real part of the wave. We should calculate the imaginary part of the wave (complex wave) because the signal of MT survey data has the phase. In order to calculate the imaginary part, we applied the Hilbert transform to the real part. Combining the real and imaginary part, complex wave in time domain is calculated. The impedance is usually calculated by Eq.2. [AB] in Eq.2 is cross spectrum in frequency domain. In this study, it is complex cross correlation in time domain. We can calculate the RFs in time domain using pole on pedestal and Hilbert transform.

$$
Z x x=\frac{\left[E_{x} \bar{H}_{x}\right]\left[H_{y} \bar{H}_{y}\right]-\left[E_{x} \bar{H}_{y}\right]\left[H_{y} \bar{H}_{x}\right]}{\left[H_{x} \bar{H}_{x}\right]\left[H_{y} \bar{H}_{y}\right]-\left[H_{x} \bar{H}_{y}\right]\left[H_{y} \bar{H}_{x}\right]}
$$

\subsection{REMOVE THE SEGMENT CONTAMINATED BY NOISE USING MEM}

In the MT processing, acquired data is usually divided into some segments. However, all segments are not analyzed since some segments are contaminated by noise. We must choose and analyze the relevant segments. Robust and coherence sort algorithm have been developed and applied. These methods are sometimes insufficient to remove the contaminated segments. Kimura et al. $(2005)^{3)}$ selects the segment by their eyes before using the coherence and robust algorithm. Calculated RFs is more accurate than only Robust and coherence algorithm. However, eyes selection is subjective, so we developed another algorithm. We use the maximum entropy method (MEM) to select the segments. We can get the power spectrum of each component (Ex or Hx etc.) by applying the MEM. We focus on the plane wave assumption of MT in order to search the contaminated segments. The same magnetic field signal penetrates at the local and remote (reference) sites, so the power spectrums of magnetic field between local and remote sites should be similar. When spectrums are compared between local and remote sites and there are some spectrum peaks at only local sites, we assume that these peaks were caused by noise and do not analyze the segment at the frequency.

\section{APPLY TO THE REAL DATA}

We apply the developed data processing to the real data acquired near Nankai trough. This data was recorded by ocean bottom electro-magnetic meter (OBEM). Fig.2 shows the raw data of each component. The strong geomagnetic variation did not occur during the observation term, so the signal was very weak and the duration of the signal was very short. We analyzed this data by developed and conventional processing. Fig.3 shows the apparent resistivity of Zxy and Zyx. The red lines were calculated by time domain processing and the blue lines were calculated by frequency domain processing. We used the software 'RRRMT' to calculate the impedance by frequency domain processing. The error amplitudes of the apparent resistivity calculated by time domain processing are much smaller than frequency domain processing. The error amplitudes calculated by frequency domain processing are between $40 \%$ and $100 \%$. On 
the other hand, it calculated by time domain processing is below $40 \%$. We can get more optimum RFs of the earth by using time domain processing. We confirm that it is difficult to get
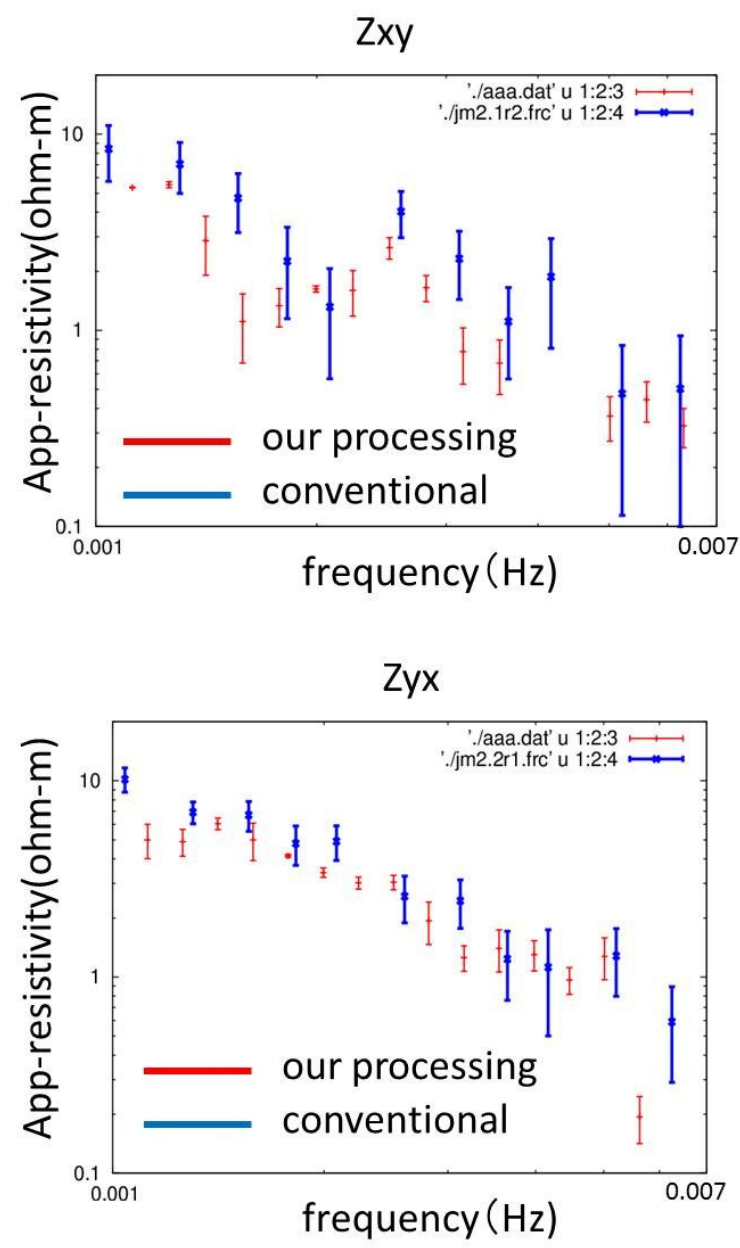

Figure 3 Apparent resistivity of Zxy and Zyx calculated by our processing (led line) and conventional process (blue line).
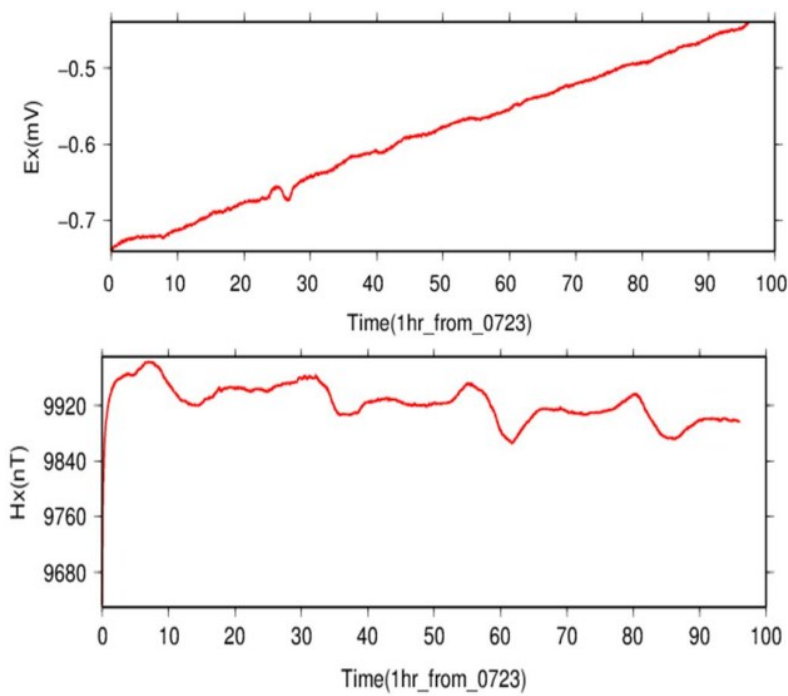

optimum RFs from the data processing based on FFT when the duration of input signal is short and $\mathrm{S} / \mathrm{N}$ ratio is low. The time domain processing by using IIR filter is efficient for analyzing the short and low $\mathrm{S} / \mathrm{N}$ data.

\section{CONCLUTION}

We developed the novel data processing in time domain using the Hilbert transform and IIR filter called pole on pedestal which extracts and amplifies the signal at a specific frequency. In addition, we apply the MEM to select the segment contaminated by noise. We applied and compared developed time domain and conventional frequency domain processing to the real data acquired at Nankai trough. The length of the segments included in the signal is very short and signal power is very small. The RFs calculated by time domain processing are more accurate than the processing based on FFT. We conclude that time domain processing using IIR filter and MEM gives us more optimum RFs when the length of data is short and $\mathrm{S} / \mathrm{N}$ ratio is low.

\section{REFERENCES}

1) Chave, A.D. and Thomson, D. J., 1989, some comments on Magnetotelluric Response Function Estimation, Journal of Geophysics Research, 94, B10, 14, 215- 225.

2) Claerbout, J.F., 1976, Fundamentals of Geophysical Data Processing: With Applications to Petroleum Processing, McFraw-Hill

3) Kimura, T., Ashida, Y., Goto, T., Kasaya, T., Mikada, H., Sanada, Y., Watanabe, T. and Yamane, K.(2005): Crustal resistivity structure around the Nankai subduction zone: Geophysical Exploration (Butsuri-Tansa), 58, 251-262
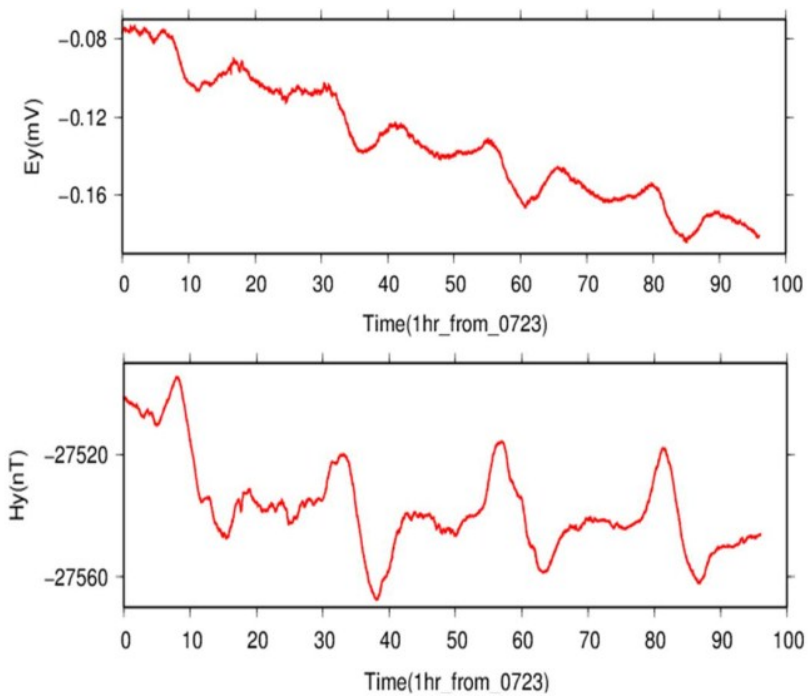

Figure 2 The raw data acquired at Nankai trough 